avances

en ciencias e ingenierías

II Simposio
Latinoamericano de Aplicaciones Nucleares en la Agricultura

\section{Eficiencia del uso de abonos verdes y urea en el cultivo de maíz de valles altos}

\author{
Yamil E. Cartagena ${ }^{1 *}$, Rafael A. Parra', Soraya P. Alvarado² ${ }^{2}$ Franklin M. Valverde' ${ }^{1}$, José L. Zambrano ${ }^{1}$ \\ 'Instituto Nacional de Investigaciones Agropecuarias (INIAP), Estación Experimental Santa Catalina, \\ Mejía, Ecuador. \\ 2Universidad Central del Ecuador (UCE), Facultad de Ciencias Agrícolas, Quito, Ecuador. \\ *Autor para correspondencia / Corresponding author, email: yamil.cartagena@iniap.gob.ec
}

Editado por /

Edited by:

Gabriela Albán

Recibido /

Received:

24/10/2020

Aceptado /

Accepted:

08/12/2020

Publicado en línea /

Published online:

01/04/2021

\section{Efficiency of the use of green manures and urea in the cultivation of corn in the highlands}

\section{Resumen}

En la actualidad, la agricultura es cada vez más dependiente de fertilizantes nitrogenados por la necesidad de producir más para satisfacer la gran demanda de alimentos en el mundo. En este sentido, la incorporación de abonos verdes puede ayudar a aumentar la bio-disponibilidad de Nitrógeno (N), elemento fundamental para el desarrollo de las plantas. El grado de aprovechamiento de N, o eficiencia del fertilizante aportado al suelo se puede medir utilizando el método convencional (diferencial) y el isotópico $\left({ }^{15} \mathrm{~N}\right)$. En este contexto, se implementó esta investigación para evaluar mediante el método convencional la eficiencia agronómica de la incorporación al suelo de abono verde de: chocho (Lupinus mutabilis), vicia (Vicia sp.) y avena (Avena sativa); con cinco niveles de nitrógeno $\left(0,45,90,135\right.$ y $\left.180 \mathrm{~kg} \mathrm{ha}^{-1}\right)$ sobre la biomasa (forraje) y el rendimiento de grano de maíz de valles altos. Adicionalmente, mediante el método isotópico ${ }^{15} \mathrm{~N}$ se evaluó la eficiencia de recuperación del $\mathrm{N}$ con la incorporación de los abonos verdes y la aplicación de $\mathrm{N}$ en tres momentos: a la siembra, a los 35 y 70 días después de la siembra (dds). En ambos experimentos se utilizó un diseño experimental de parcela dividida, donde la parcela grande correspondió al cultivo incorporado al suelo y en las parcelas pequeñas los niveles o tiempo de aplicación del nitrógeno, con tres repeticiones. La incorporación de abonos verdes no produjo ningún efecto significativo sobre el rendimiento de maíz, excepto en el ensayo isotópico, donde la incorporación de chocho incrementó significativamente la producción de follaje a 22.69 t ha $^{-1}$. El mayor rendimiento de grano de maíz (6.76 t ha-1) se obtuvo con la dosis de $135 \mathrm{~kg} \mathrm{ha}^{-1}$ de nitrógeno, diferenciándose estadísticamente de la dosis de 0 y $45 \mathrm{~kg} \mathrm{ha}^{-1}$. El método isotópico con el uso de ${ }^{15} \mathrm{~N}$ permitió diferenciar los niveles de absorción de nitrógeno entre las épocas de aplicación, observándose que la mayor absorción de este elemento y la mayor eficiencia de la fertilización nitrogenada se obtuvieron a los 70 dds (V6-V7). Estos resultados demuestran la importancia de las técnicas isotópicas para estudios de nutrición vegetal y permitirá ajustar las recomendaciones de fertilización nitrogenada para el cultivo de maíz de la sierra del Ecuador.

Palabras clave: Maíz, Nitrógeno 15, Isótopos, Rendimiento. 


\begin{abstract}
Currently, agriculture is increasingly dependent on nitrogenous fertilizers due to the need to produce more to satisfy the great food demand in the world. In this sense, incorporation of green manures can help to increase the bioavailability of Nitrogen $(\mathrm{N})$, a fundamental element for the development of plants. The $\mathrm{N}$ use efficiency can be measured using the conventional (differential) and the isotopic $\left({ }^{15} \mathrm{~N}\right)$ method. In this context, a research was implemented to evaluate the effect of two green manure species: (Lupinus mutabilis and Vicia sp.) and a cereal species (Avena sativa); and five nitrogen levels $\left(0,45,90,135\right.$ and $\left.180 \mathrm{~kg} \mathrm{ha}^{-1}\right)$ on the biomass (forage) and grain yield of corn in the highlands of Ecuador. Additionally, efficiency of the nitrogen fertilizer was evaluated at three times: at sowing, 35 and 70 days after sowing (das). In both experiments, a split plot design was used, where the large plot contained the crop incorporated into the soil, and the small plots had the levels of $\mathrm{N}$ or application time, with three replications. Incorporation of green manures did not produce any significant effect on corn yield, except in the isotopic test, where the incorporation of lupine significantly increased the production of forage to $22.69 \mathrm{t} \mathrm{ha}^{-1}$. The highest yield of corn grain $\left(6.76 \mathrm{t} \mathrm{ha}^{-1}\right)$ was obtained with dose of $135 \mathrm{~kg} \mathrm{ha}^{-1}$ of $\mathrm{N}$, differing statistically from the dose of 0 and 45 $\mathrm{kg} \mathrm{ha}^{-1}$. The isotopic method with the use of ${ }^{15} \mathrm{~N}$ allowed to differentiate the levels of $\mathrm{N}$ absorption among the application times, observing that the highest absorption of this element and the highest efficiency of $\mathrm{N}$ occurred at 70 das (V6-V7). These results demonstrate the importance of isotopic techniques for plant nutrition studies and will allow to adjust the nitrogen fertilization recommendations for the cultivation of corn in the Ecuadorian highlands.
\end{abstract}

Keywords: Maize, Nitrogen 15, Isotopes, Yield.

\title{
INTRODUCCIÓN
}

El Nitrógeno (N) es uno de los elementos que requiere la planta en grandes cantidades seguido por el Fósforo (P) y el Potasio (K). El N está presente en procesos metabólicos de suma importancia para el desarrollo de la planta directamente relacionado con la formación de ácidos nucleicos y proteínas, además de ser un componente fundamental de la clorofila que es vital para la fotosíntesis [1].

En la actualidad, la agricultura es cada vez más dependiente de fertilizantes y especialmente de los nitrogenados por la necesidad de producir más para satisfacer la gran demanda de alimentos en el mundo [2]. En el mercado de los fertilizantes nitrogenados se puede apreciar la gran demanda que existe por el monofosfato de amonio (MAP) (11-52-0), difosfato de amonio (DAP) (18-46-0), nitrato de amonio (330-0), sulfato de amonio (21-0-0-24 S) y urea (46-0-0); ésta última supera en demanda a todos los fertilizantes juntos antes mencionados, siendo el Ecuador muy dependiente de la importación de estos fertilizantes [3, 4].

La eficiencia del uso del $\mathrm{N}$ se refiere a la cantidad de $\mathrm{N}$ que la planta absorbió del fertilizante que fue aplicado al suelo. En el cultivo de maíz se ha observado mediante la 
experimentación que un 34\% del $\mathrm{N}$ aplicado al suelo fue absorbido por la planta. Esta eficiencia del $\mathrm{N}$ se obtuvo en sistemas de producción convencionales y en aquellos con cero labranza y quema de rastrojo [5]. La eficiencia agronómica del uso del N refiere al rendimiento del cultivo en relación a la cantidad de nitrógeno aplicado, habiéndose reportado eficiencias agronómicas de hasta $23 \%$ en un híbrido de maíz evaluado en zonas bajas el Litoral ecuatoriano [6].

El N y su eficiencia han recibido especial atención por ser el nutriente más móvil dentro de la relación atmósfera, suelo y planta. El grado de aprovechamiento de $\mathrm{N}$ del fertilizante aportado al suelo ha sido medido utilizando diferentes métodos: el diferencial (convencional) y el isotópico. El primero, el más sencillo y menos costoso, se basa en estimar la diferencia de $\mathrm{N}$ absorbido entre las plantas fertilizadas y las no fertilizadas. Puede ocurrir que las plantas fertilizadas absorban más $N$ del suelo que las plantas testigo, debido a un mayor desarrollo radical inducido por la fertilización. El método isotópico utiliza ${ }^{15} \mathrm{~N}$ y permite conocer la cantidad de $\mathrm{N}$ absorbido por la planta que vino del fertilizante aplicado, bajo el supuesto que no se produce intercambio isotópico entre el $\mathrm{N}$ del fertilizante y el del suelo $[7,8]$.

Se conoce que el fraccionamiento de las aplicaciones de $\mathrm{N}$ durante el ciclo de cultivo permite incrementar la eficiencia del uso del fertilizante y consecuentemente las cosechas; ya que la planta recibe lo que necesita a medida que aumenta su desarrollo, en lugar de realizar una sola aplicación antes de la siembra, práctica con la que la mayor parte del fertilizante no es aprovechado por la planta [9].

La práctica de la agricultura sin la utilización de fertilizantes nitrogenados se hace imposible cuando se requiere la generación de alimentos en gran cantidad para una población que crece cada día; sin embargo, es posible la utilización de nuevas tecnologías como la incorporación de abonos verdes, siembra de leguminosas, adiciones constantes de materia orgánica, mismas que ayudan a aumentar la bio-disponibilidad de N [10].

El uso de abonos verdes mediante el empleo de especies leguminosas es una técnica que aporta $\mathrm{N}$ al suelo y al cultivo siguiente de manera considerable, el $\mathrm{N}$ que aportan las leguminosas es fijado principalmente de la atmósfera, debido a la capacidad de fijación biológica que poseen mediante la simbiosis con baterías Rhizobium spp [11]. Se estima que la incorporación de abonos verdes al suelo permite incorporar entre 50 a $80 \mathrm{~kg} \mathrm{~N} \mathrm{ha}^{-1}$ para la avena (Avena sativa) mientras que para la vicia la cantidad está entre 150 y $250 \mathrm{~kg}$ ha $^{-1}$. Para el caso del frijol (Phaseolus vulgaris) el aporte es de 30 a $60 \mathrm{~kg} \mathrm{~N} \mathrm{ha}^{-1}$, para el cultivo de chocho (Lupinus mutabilis) la cantidad es mayor siendo entre 300 a $600 \mathrm{~kg} \mathrm{~N} \mathrm{ha}^{-1}$ [12].

La materia orgánica del suelo es la principal fuente de N para las plantas. De esta se derivan el nitrato y el amonio. Una vez que el nitrato es absorbido por la planta, es reducido con un gasto energético que proviene de la fotosíntesis. En cambio, el amonio no se reduce y se incorpora inmediatamente a las aminas y amidas de la planta [7]. El objetivo de esta investigación fue evaluar la eficiencia agronómica del uso de abonos verdes y la fertilización nitrogenada sobre la biomasa y el rendimiento del cultivo de maíz de valles altos (>2000 msnm), y estimar la mejor época de aplicación de nitrógeno que permita incrementar rendimiento y la eficiencia de recuperación del nitrógeno aplicado en el cultivo de maíz de altura. 


\section{MATERIALES Y MÉTODOS}

El experimento se realizó en el Campo Académico Docente Experimental La Tola (CADET) de la Facultad de Ciencias Agrícolas de la Universidad Central del Ecuador (UCE), cuya ubicación política, geográfica y clima, se presenta en la Tabla 1. Las propiedades químicas y físicas del suelo donde se realizó la investigación se detallan en la Tabla 2.

TABLA 1. Ubicación política, geográfica y clima del Campo Académico Docente Experimental La Tola (CADET), Pichincha.

\begin{tabular}{|c|c|}
\hline Ubicación & Localidad \\
\hline Provincia & Pichincha \\
Cantón & Quito \\
Parroquia & Tumbaco \\
Sitio & CADET \\
\hline Altitud (m.s.n.m.) & 2460 \\
\hline Latitud & $78^{\circ} 21^{\prime} 18^{\prime \prime} \mathrm{S}$ \\
\hline Longitud & $00^{\circ} 13^{\prime} 49^{\prime \prime} \mathrm{O}$ \\
Temperatura ( $\left.^{\circ} \mathrm{C}\right)$ & 15 \\
\hline Precipitación (mm) & 927 \\
\hline Humedad relativa (\%) & 76 \\
\hline Heliofania (h) & 1957 \\
\hline Nubosidad (octas) & 6 \\
\hline
\end{tabular}

Fuente: [28].

TABLA 2. Reporte del análisis químico de suelos donde se evaluó el efecto del uso de abonos verdes, dosis y tiempo de aplicación de N en el cultivo de maíz de valles altos. Campo Académico Docente Experimental La Tola, Pichincha.

\begin{tabular}{|c|c|c|c|}
\hline Nutriente & Valor & Interpretación & Unidad \\
\hline $\mathrm{pH}$ & 6.40 & LA & \\
\hline $\mathrm{MO}$ & 3.00 & M & $\%$ \\
\hline $\mathrm{N}-\mathrm{NH}_{4}^{+}$ & 31.00 & M & $\mathrm{mg} \mathrm{kg}^{-1}$ \\
\hline P & 18.00 & M & $\mathrm{mg} \mathrm{kg}^{-1}$ \\
\hline S & 13.00 & M & $\mathrm{mg} \mathrm{kg}^{-1}$ \\
\hline K & 0.65 & A & meq $100 \mathrm{ml}^{-1}$ \\
\hline $\mathrm{Ca}$ & 7.30 & M & meq $100 \mathrm{ml}^{-1}$ \\
\hline $\mathrm{Mg}$ & 3.60 & A & meq $100 \mathrm{ml}^{-1}$ \\
\hline $\mathrm{Zn}$ & 0.30 & B & $\mathrm{mg} \mathrm{kg}^{-1}$ \\
\hline $\mathrm{Cu}$ & 6.70 & A & $\mathrm{mg} \mathrm{kg}^{-1}$ \\
\hline $\mathrm{Fe}$ & 78.00 & A & $\mathrm{mg} \mathrm{kg}^{-1}$ \\
\hline $\mathrm{Mn}$ & 9.50 & M & $\mathrm{mg} \mathrm{kg}^{-1}$ \\
\hline
\end{tabular}




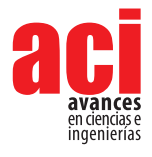

\begin{tabular}{|c|c|c|c|}
\hline Nutriente & \multicolumn{1}{|c|}{ Valor } & Interpretación & Unidad \\
\hline B & 0.90 & $\mathrm{~B}$ & $\mathrm{mg} \mathrm{kg}^{-1}$ \\
\hline Textura & Franco Arenoso & & \\
\hline
\end{tabular}

$A=A l t o, M=$ Medio, $B=$ Bajo y $L A=L$ igeramente ácido.

\section{Determinación de la eficiencia agronómica en la incorporación de abonos verdes $\mathrm{y}$ dosis de $\mathrm{N}$}

En el CADET se implementó un experimento para evaluar el efecto de tres especies de abono verde incorporadas al suelo. Se sembró dos especies leguminosas fijadoras de nitrógeno como chocho (Lupinus mutabilis) y vicia (Vicia sp.) y se utilizó la avena (Avena sativa), como especie no fijadora de nitrógeno y fue el testigo. Se incorporaron como biomasa luego de la floración $\left(6.44,6.87\right.$ y 7.49 t ha $^{-1}$ de chocho, vicia y avena, respectivamente). En el siguiente ciclo de cultivo se sembró maíz de valles altos con cinco niveles de nitrógeno $\left(0,45,90,135\right.$ y $\left.180 \mathrm{~kg} \mathrm{ha}^{-1}\right)$, siendo urea (46\% de N) la fuente del fertilizante utilizado. El fertilizante nitrogenado se aplicó fraccionadamente en partes iguales a la siembra, 35 y 70 días después de la siembra; que corresponden a las etapas vegetativas del maíz V3-V4 y V6-V7, respectivamente.

El diseño experimental utilizado fue de parcela dividida [13], con 15 tratamientos en total. La parcela grande (208 m², 26 surcos de 10 m de largo) correspondió a las especies de leguminosas y al cereal incorporados, y en las parcelas pequeñas $\left(16 \mathrm{~m}^{2}, 5\right.$ surcos de $4 \mathrm{~m}$ de largo) los niveles de nitrógeno, con tres repeticiones.

El rendimiento de la biomasa seca se determinó en la etapa R3 (madurez fisiológica), en la cual se incluyó los órganos vegetativos y reproductivos de la planta de maíz. El rendimiento de grano se evaluó a la cosecha en seco, pesando las mazorcas cosechadas luego de la madurez fisiológica, y ajustando el rendimiento del grano al 14\% de humedad [14].

La eficiencia agronómica del uso del N (EAN) se estimó mediante la diferencia entre el rendimiento del tratamiento con fertilizante y el rendimiento del tratamiento testigo (sin fertilizante), dividida para la cantidad de nitrógeno aplicado y el resultado se expresó en porcentaje $[6,15]$.

La absorción de $\mathrm{N}$ se la estimó en toda la planta, para lo cual se tomaron cuatro plantas del surco central por cada parcela. Luego de un proceso de picado y secado en estufa a $60^{\circ} \mathrm{C}$, la muestra se analizó mediante el método de semimicro-Kjeldahl [16] en el laboratorio del Departamento de Manejo de Suelos y Aguas de la Estación Experimental Santa Catalina del INIAP.

\section{Eficiencia de la recuperación de $\mathrm{N}$ en la incorporación de abonos verdes y el tiempo de aplicación del fertilizante nitrogenado, mediante la técnica isotópica ${ }^{15} \mathrm{~N}$}

En un diseño de parcela dividida con tres repeticiones, se evaluó la eficiencia de la incorporación de abonos verdes (las mismas especies indicadas en el experimento 
anterior) y la aplicación de $\mathrm{N}$ (dosis de $90 \mathrm{~kg} \mathrm{ha}^{-1}$ ) en tres épocas de aplicación: siembra, 35 y 70 días después de la siembra. El tamaño de las parcelas fue el mismo que se utilizó en el experimento anterior. Los tratamientos correspondientes a las épocas de aplicación se ubicaron en las parcelas pequeñas. El fertilizante marcado fue urea ${ }^{15} \mathrm{~N}$, que se aplicó según los tratamientos en estudio (Tabla 3). La urea marcada con ${ }^{15} \mathrm{~N}$ (concentración, $5.16 \%$ átomos en exceso) se aplicó diluida en agua destilada y con regadera a lo largo de los surcos de la parcela pequeña.

TABLA 3. Tratamientos con especies leguminosas y avena incorporados al suelo previo al cultivo de maíz, con diversas épocas de aplicación de nitrógeno utilizando la técnica isotópica de ${ }^{15} \mathrm{~N}$.

\begin{tabular}{|c|c|c|c|c|}
\hline \multirow[t]{2}{*}{ Tratamientos } & \multirow{2}{*}{$\begin{array}{c}\text { Abono verde incorporado al suelo } \\
\text { (parcela grande) }\end{array}$} & \multicolumn{3}{|c|}{$\begin{array}{l}\text { Época y dosis N } \\
\left(\mathrm{kg} \mathrm{ha}^{-1}\right)\end{array}$} \\
\hline & & Siembra & $35 \mathrm{ddds}^{t}$ & 70 dds \\
\hline $\mathrm{T} 1$ & Chocho & $30^{*}$ & 30 & 30 \\
\hline $\mathrm{T} 2$ & Chocho & 30 & $30^{*}$ & 30 \\
\hline $\mathrm{T} 3$ & Chocho & 30 & 30 & $30^{*}$ \\
\hline $\mathrm{T} 4$ & Vicia & $30^{*}$ & 30 & 30 \\
\hline $\mathrm{T} 5$ & Vicia & 30 & $30^{*}$ & 30 \\
\hline T6 & Vicia & 30 & 30 & $30^{*}$ \\
\hline $\mathrm{T7}$ & Avena & $30^{*}$ & 30 & 30 \\
\hline T8 & Avena & 30 & $30^{*}$ & 30 \\
\hline T9 & Avena & 30 & 30 & $30^{*}$ \\
\hline $\mathrm{T} 10$ & Control sin abono verde & $30^{*}$ & 30 & 30 \\
\hline
\end{tabular}

Para la siembra de ambos experimentos se utilizó la distancia de siembra de $0.80 \mathrm{~m}$ entre surcos y $0.25 \mathrm{~m}$ entre plantas, obteniéndose una densidad de población de 50000 plantas ha-1. Se utilizó semilla de maíz (Zea mays, L.) de la variedad INIAP-180 [17]. Los experimentos se sembraron el 24 de mayo del 2016 (45 días después de la incorporación del abono verde) y se cosechó en seco (a la madurez fisiológica) el 31 de enero del 2017. Se realizaron cuatro aplicaciones de riego de auxilio con láminas de 4 mm cada una, mientras que el resto del manejo agronómico se lo realizó según las recomendaciones técnicas para cultivar maíz en la sierra del Ecuador [18].

Para evaluar la eficiencia de uso del fertilizante (EUN), según la metodología isotópica $\left(E U^{15} \mathrm{~N}\right)$, se determinó la cantidad del nitrógeno absorbido por la planta del tratamiento con fertilizante marcado con ${ }^{15} \mathrm{~N}$, y este resultado se dividió entre la dosis de nitrógeno aplicado y el resultado se expresó en porcentaje [19]. Para el análisis isotópico de ${ }^{15} \mathrm{~N}$, se enviaron muestras secas, molidas y envasadas a la Universidad de Florida, para su determinación por la técnica de espectrometría de masas [20]. La EUN total se estimó sumando la eficiencia obtenida en las tres aplicaciones, realizando el ANOVA solo para los tratamientos de la parcela grande (incorporación de abonos verdes). 


\section{Análisis estadísticos}

En ambos experimentos se realizaron Análisis de Varianza (ANOVA) para todas las variables evaluadas y en caso de existir diferencias estadísticas significativas $(p<0,05)$ se realizó la prueba de Fisher LSD para diferencia de medias. Previo al ANOVA, los datos de cada variable fueron sometidos a la prueba de normalidad de Shapiro-Wilks, y en caso de datos no normalizados se procedió con la transformación logarítmica de los mismos. Se utilizó el programa estadístico Infostat [21].

\section{RESULTADOS}

\section{Determinación de la eficiencia agronómica de la incorporación de abonos verdes y dosis de nitrógeno}

El análisis de la varianza para la absorción de $N$, encontró diferencias altamente significativas para niveles de nitrógeno $(p<0.01)$ y no tuvieron significancia estadística las especies leguminosas incorporadas y la interacción. El coeficiente de variación del $12.63 \%$ es confiable para esta investigación (Tabla 4). La prueba de LSD al 5\% para la extracción de nitrógeno mostró tres rangos de significancia en las dosis de nitrógeno, encontrándose la menor extracción en el tratamiento $\sin \mathrm{N}$ (dosis de $0 \mathrm{~kg} \mathrm{ha}^{-1}$ ) con $173.36 \mathrm{~kg} \mathrm{ha}^{-1}$ de N, y la mayor extracción con $233.84 \mathrm{~kg} \mathrm{ha}^{-1}$ de N para la dosis más alta del fertilizante (180 kg ha-1). La absorción de nitrógeno con las especies de incorporadas lupinos, vicia y avena en el cultivo de maíz fueron similares (Tabla 4).

TABLA 4. Promedios y significación estadística del ADEVA de la absorción de nitrógeno (N) y eficiencia agronómica de la fertilización nitrogenada (EAN) utilizando la técnica convencional no isotópica en el cultivo de maíz de valles altos para diversos tipos de abonos verdes y dosis de $\mathrm{N}$.

\begin{tabular}{|c|c|c|c|c|}
\hline $\begin{array}{c}\text { Abono verde (A)/ Dosis } \\
\mathrm{N}(\mathrm{D})\left(\mathrm{kg} \mathrm{ha}^{-1}\right)\end{array}$ & $\begin{array}{l}\text { Absorción } \\
\mathrm{N}\left(\mathrm{kg} \mathrm{ha}^{-1}\right)\end{array}$ & $\begin{array}{c}\text { Biomasa } \\
\left(\text { tha }^{-1}\right)\end{array}$ & $\begin{array}{l}\text { Rendimiento grano } \\
\text { (t } \text { ha }^{-1} \text { ) }\end{array}$ & $\begin{array}{c}\text { EAN } \\
\left(\mathrm{kg} \mathrm{kg} \mathrm{N}^{-1}\right)\end{array}$ \\
\hline Chocho & 231.78 & 20.17 & 6.06 & 19.74 \\
\hline Vicia & 231.23 & 19.88 & 5.91 & 17.39 \\
\hline Avena & 222.61 & 19.03 & 5.99 & 19.36 \\
\hline 0 & $173.36 \mathrm{a}^{\dagger}$ & $16.32 \mathrm{a}$ & $4.14 \mathrm{a}$ & - \\
\hline 45 & $229.77 b$ & $19.42 b$ & $6.12 b$ & 39.99 a \\
\hline 90 & $244.42 b c$ & $21.35 c$ & $6.55 b c$ & $24.82 b$ \\
\hline 135 & $261.29 c$ & $21.34 c$ & $6.76 c$ & $18.08 \mathrm{c}$ \\
\hline 180 & $233.84 b$ & $20.05 b c$ & $6.35 b c$ & $11.25 d$ \\
\hline Sig. $\operatorname{ADEVA}^{\ddagger}(\mathrm{A})$ & Ns & Ns & Ns & Ns \\
\hline Sig. ADEVA (D) & $* *$ & ** & $* *$ & $* *$ \\
\hline Sig. ADEVA (AxD) & Ns & Ns & Ns & Ns \\
\hline CV (\%) & 12.63 & 8.31 & 8.10 & 33.01 \\
\hline
\end{tabular}

${ }^{\dagger}$ Medias seguidas con una letra común no son significativamente diferentes según LSD Fisher ( $\left.p>0.05\right)$.

${ }^{\ddagger} \mathrm{Ns}=$ no significativo, ${ }^{* *}=$ altamente significativo $(p<0,001)$. 
El análisis de la varianza para la biomasa de las especies incorporadas de leguminosas y cereal, encontró diferencias altamente significativas para las dosis de nitrógeno ( $p<$ 0.01) y no tuvieron significación estadística las especies incorporadas y la interacción. El coeficiente de variación fue de $8.31 \%$. La prueba de LSD al 5\% para la biomasa de maíz con las dosis de nitrógeno encontró tres rangos de significancia, ubicándose con el mayor valor la dosis de $180 \mathrm{~kg} \mathrm{ha}^{-1}$ de N con $20.05 \mathrm{t} \mathrm{ha}^{-1}$ de biomasa o forraje (Tabla 4 y Figura 1).

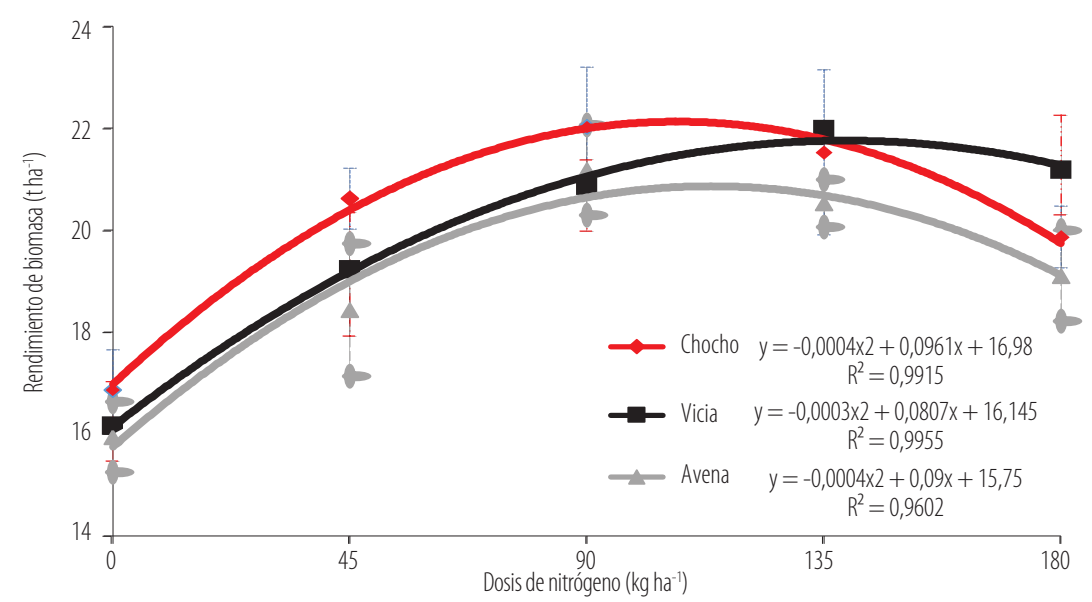

FIGURA 1. Efecto de las dosis de nitrógeno y la incorporación de abonos verdes en el rendimiento de biomasa en el cultivo de maíz de valles altos. Las barras representan el error estándar de la media.

El análisis de la varianza para el rendimiento de grano tuvo diferencias significativas para dosis de nitrógeno $(p<0.001)$ y no se encontró diferencias estadísticas por especies incorporadas y su interacción. El coeficiente de variación fue de $8.10 \%$. La prueba de significación de LSD al 5\%, tuvo tres rangos de significación, siendo el mayor la dosis de $135 \mathrm{~kg} \mathrm{ha}^{-1}$ con 6.55 t ha-1 (Tabla 4).

El análisis de la varianza para la EAN, encontró diferencias altamente significativas para las dosis de nitrógeno; en tanto que, para las especies incorporadas y la interacción, no se encontraron diferencias estadísticas. Se tuvo un coeficiente de variación de $34.01 \%$. La prueba de LSD al 5\%, encontró tres rangos de significación, el mayor valor encontrado fue de $39.99 \mathrm{~kg}$ de grano $\mathrm{kg} \mathrm{N}^{-1}$ con la dosis de $45 \mathrm{~kg} \mathrm{ha}^{-1}$ (Tabla 4).

La dosis de nitrógeno aplicadas en el maíz con las especies incorporadas, tuvieron un efecto cuadrático, notándose que los mayores rendimientos se encuentran entre las dosis de 90 y $135 \mathrm{~kg} \mathrm{ha}^{-1}$ de nitrógeno (Figura 1).

\section{Eficiencia de la recuperación de $\mathrm{N}$ en la incorporación de abonos verdes y el tiempo de aplicación del fertilizante nitrogenado, mediante la técnica isotópica ${ }^{15} \mathrm{~N}$}

El análisis de la varianza para la concentración de ${ }^{15} \mathrm{~N}$ no encontró diferencias estadísticas para la incorporación de las especies de leguminosas y cereal como abono verde. Sin embargo, se encontró alta significación estadística para el tiempo 
de aplicación y ninguna significación estadística para la interacción entre tipo de abono verde y tiempo de aplicación. El coeficiente de variación fue de $4.92 \%$. La prueba de LSD al 5\% para el tiempo de aplicación del nitrógeno encontró tres rangos de significación, siendo la concentración más alta con $0.56 \%$ de ${ }^{15} \mathrm{~N}$ a los 70 días después de la siembra (Tabla 5).

El análisis de la varianza para la concentración de $\mathrm{N}$ total, no tuvo significación estadística para la aplicación del abono verde, tiempo de aplicación y la interacción. El coeficiente de variación fue de 34.14\% (Tabla 5).

TABLA 5. Promedios y significación estadística del ADEVA de la concentración de nitrógeno (N) y de la eficiencia de uso de la fertilización nitrogenada (EUN) utilizando la técnica isotópica ${ }^{15} \mathrm{~N}$ en el cultivo de maíz de valles altos para diversos tipos de abonos verdes y tiempo de aplicación de urea.

\begin{tabular}{|c|c|c|c|c|c|c|}
\hline $\begin{array}{l}\text { Abono verde } \\
\text { (A)/Tiempo de } \\
\text { aplicación(T) }\end{array}$ & $\begin{array}{c}\text { Concentración } \\
{ }^{15} \mathrm{~N} \\
(\%)\end{array}$ & $\begin{array}{c}\text { Concentración } \\
\text { Ntotal } \\
(\%)\end{array}$ & $\begin{array}{l}\text { Biomasa } \\
\text { (tha-1) }\end{array}$ & $\begin{array}{c}\text { Rendimiento } \\
\text { grano } \\
\text { (tha-1) }\end{array}$ & $\begin{array}{l}\text { EU15N } \\
(\%)\end{array}$ & $\begin{array}{c}\text { EU15 } \\
\text { acumulado } \\
(\%)\end{array}$ \\
\hline Chocho & 0.53 & 1.09 & $22.69 a$ & 8.41 & 14.29 & 42.86 \\
\hline Vicia & 0.52 & 1.12 & $22.04 a b$ & 7.56 & 13.08 & 39.24 \\
\hline Avena & 0.53 & 0.86 & $21.58 \mathrm{~b}$ & 8.16 & 10.32 & 30.94 \\
\hline Sin residuo & 0.52 & 0.87 & $19.63 \mathrm{~b}$ & 8.27 & 8.64 & 25.91 \\
\hline $0 d d s^{\dagger}$ & $0.50 a^{\ddagger}$ & 0.96 & 21.48 & 8.08 & $8.92 \mathrm{a}$ & \\
\hline $45 \mathrm{dds}$ & $0.53 b$ & 1.05 & 22.83 & 7.99 & $13.80 a b$ & 37.59 \\
\hline $70 \mathrm{dds}$ & $0.56 c$ & 1.03 & 21.40 & 8.13 & $14.87 \mathrm{~b}$ & \\
\hline Sig. ADEVA' (A) & Ns & Ns & * & Ns & Ns & Ns \\
\hline Sig. ADEVA (T) & $* *$ & Ns & Ns & Ns & * & Ns \\
\hline Sig. ADEVA (AxT) & Ns & Ns & Ns & Ns & Ns & Ns \\
\hline CV (\%) & 4.92 & 34.14 & 9.82 & 16.38 & 18.22 & 9.92 \\
\hline
\end{tabular}

¥Medias seguidas con una letra común no son significativamente diferentes según Lsd Fisher ( $p>0.05)$. ${ }^{\dagger}$ dds = días después de la siembra, 45 dds (etapa vegetativa del maíz V3-V4), 70 dds (etapa vegetativa V6-V7). 'Ns = no significativo, ${ }^{*}=$ significativo $(p<0.05), y^{* *}=$ altamente significativo $(p<0,001)$.

El análisis de la varianza para la biomasa tuvo significación estadística para la aplicación de abono verde y no tuvo significancia estadística para tiempo de aplicación y la interacción. La prueba de significación del LSD al 5\% obtuvo dos rangos de significación, siendo el mayor el cultivo de chocho con una biomasa de 22.69 t ha $^{-1}$ (Tabla 5).

El análisis de la varianza para el rendimiento de grano no encontró significación estadística para la aplicación del abono verde, tiempo de aplicación y la interacción. El coeficiente de variación fue de 16.38\% (Tabla 5).

El análisis de la varianza para la eficiencia de uso de ${ }^{15} \mathrm{~N}\left(\mathrm{EU}^{15} \mathrm{~N}\right)$, mostró significación estadística para el tiempo de aplicación de nitrógeno y no encontró significación estadística para la aplicación de abono verde y la interacción. El coeficiente de variación del 18.22\%. La prueba de LSD al 5\% para el tiempo de aplicación del nitrógeno obtuvo dos rangos, siendo el mejor a los 70 días después de la siembra con 14.87\% (Tabla 5). 
El análisis de la varianza para la eficiencia de uso acumulado de ${ }^{15} \mathrm{~N}\left(E U^{15} \mathrm{~N}\right.$ acumulado), no tuvo significación estadística para la aplicación del abono verde, tiempo de aplicación y la interacción. El coeficiente de variación fue de 9.99\% (Tabla 5).

\section{DISCUSIÓN}

La cantidad incorporada de abono verde está en función de la especie empleada, desarrollo vegetativo, tipo de suelo, condiciones climáticas y demás factores de la producción. La mejor época para realizar la incorporación del abono verde es en la floración de las especies debido a que en este estado los nutrientes están concentrados en los órganos vegetativos y aún no han sido trasladados hacia otros órganos como frutos, por lo que los nutrientes serán aprovechados en su totalidad [11].

La acumulación y re-movilización de $\mathrm{N}$ en la planta de maíz se expresa con el índice de cosecha, el cual tiene valores que varían entre 59 y 69\%, considerando la relación fuente-demanda. Se estima que entre el 31 y $41 \%$ del N acumulado en la biomasa se queda en el rastrojo y es devuelto al suelo mediante la incorporación [7].

Otros estudios han reportado un efecto de la incorporación de abono verde sobre el rendimiento del cultivo $[22,23]$. En este estudio no se observó un efecto significativo de la incorporación de abonos verdes, tanto en el método convencional como isotópico (Tabla 4). Esto pudo deberse a varios factores, como la disponibilidad de nitrógeno en el suelo donde se realizó el experimento (Tabla 2) y la incorporación del abono verde que fue muy cerca de la siembra (45 días); lo que no permitió que el nitrógeno incorporado por el abono esté disponible para el cultivo de maíz debido a los periodos de mineralización, que por las condiciones de altitud y temperatura se alargan en la Sierra del Ecuador [24].

Se observó un efecto significativo sobre el rendimiento del cultivo de maíz, en biomasa y de grano, con la aplicación del nitrógeno utilizando como fuente la urea. Estos resultados corroboran lo indicado por $[25,26]$, quienes reportaron un incremento de rendimiento de hasta $10 \%$ sobre el testigo. En este estudio, el mayor rendimiento de grano se observó con la dosis de $135 \mathrm{~kg} \mathrm{ha}^{-1}$ con $6.76 \mathrm{t} \mathrm{ha}^{-1}$ (grano seco, ajustado al $14 \%$ de humedad), lo que representó un incremento de $39 \%$ en referencia al testigo sin aplicación de nitrógeno (Tabla 4).

La eficiencia agronómica del nitrógeno (EAN) en el cultivo de maíz de altura fue mayor con la menor dosis de nitrógeno (45 $\mathrm{kg} \mathrm{N} \mathrm{ha}^{-1}$ ), que correspondió a un rendimiento de 6.12 t ha $^{-1}$, lo que significa que con cada kilogramo de N aplicado se obtuvo 39.99 kg de maíz. Zamudio y colaboradores [27] reportaron EAN de $20.9 \mathrm{~kg}$ de grano $\mathrm{kg} \mathrm{de} \mathrm{N}^{-1}$, con una dosis de $168 \mathrm{~kg} \mathrm{~N} \mathrm{ha}^{-1}$, que fue similar a la EAN obtenida en este estudio con la dosis de $180 \mathrm{~kg} \mathrm{~N} \mathrm{ha}^{-1}$ (Tabla 4 y Figura 2). 


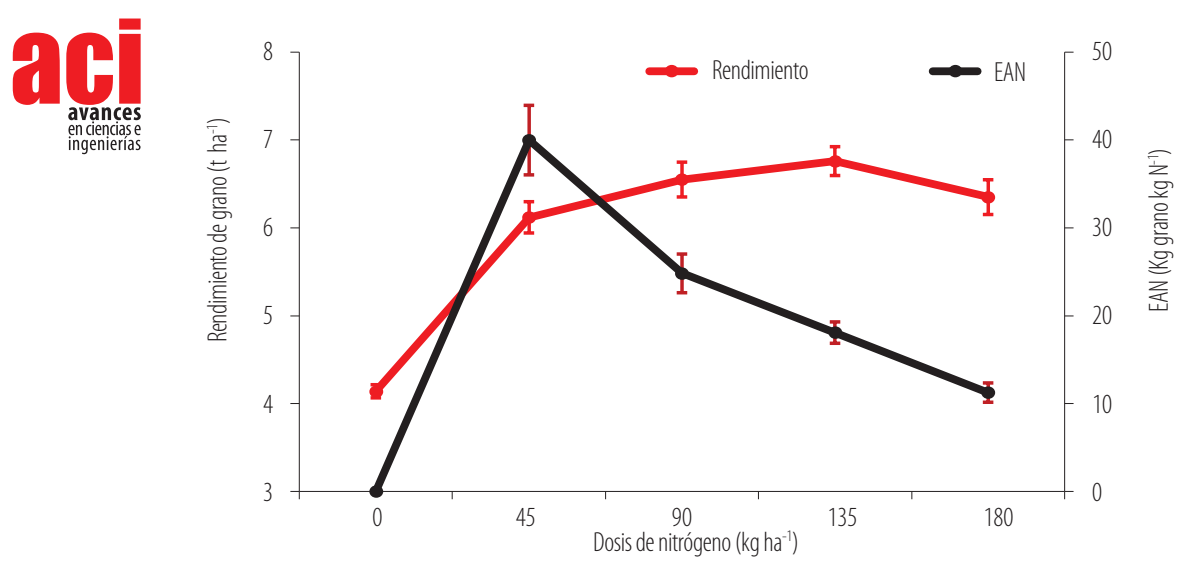

FIGURA 2. Efecto de cinco dosis de nitrógeno en el rendimiento de grano y la eficiencia agronómica del uso del nitrógeno (EAN) en el cultivo de maíz de valles altos. Las barras representan la desviación estándar de la media.

El uso de ${ }^{15} \mathrm{~N}$ permitió encontrar diferencias estadísticas significativas para el tiempo de aplicación del nitrógeno en el cultivo de maíz de altura, mientras que con el uso de la técnica convencional de $\mathrm{N}$ total no fue posible (Tabla 5). Este estudio reportó que la mayor absorción de nitrógeno (ER $\left.{ }^{15} \mathrm{~N}\right)$ se obtuvo a los 70 dds, con una eficiencia de $14.87 \%$. Este resultado es diferente a la recomendación que realiza el Programa de Maíz del INIAP, que indica que el fertilizante se debe aplicar a la siembra y a los 45 días después de la siembra [18]. Esto implica que se deben revisar las recomendaciones sobre el tiempo de aplicación de la fertilización nitrogenada para el cultivo de maíz de altura.

La eficiencia de uso de la fertilización nitrogenada EU ${ }^{15} \mathrm{~N}$ acumulada para la incorporación de abono verde en el cultivo de maíz de altura fue de 37.68\%, 11.77\% superior al testigo donde no se incorporó materia verde. Estos valores son muy similares a los reportados por otros autores [19].

\section{CONCLUSIONES}

La incorporación de abono verde (lupino) incrementó la biomasa (follaje) en el cultivo de maíz en un 15\% con respecto al testigo; pero no influyó en el rendimiento de

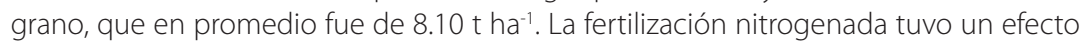
significativo sobre el rendimiento del cultivo. El mayor rendimiento de grano y follaje se observó con la dosis de $135 \mathrm{~kg} \mathrm{~N} \mathrm{ha}^{-1}$; mientras que la mayor eficiencia agronómica (EAN) se obtuvo con la dosis de $45 \mathrm{~kg} \mathrm{~N} \mathrm{ha}^{-1}$, lo que significa que con cada kilogramo de $\mathrm{N}$ aplicado se obtuvo $39.99 \mathrm{~kg}$ de maíz.

La técnica isotópica permitió diferenciar la época en la que el cultivo de maíz de valles altos de la región Andina del Ecuador realiza la mejor absorción de nitrógeno, observándose que la mayor eficiencia en el uso de nitrógeno (EUN) con 14.87\% que se logró a los 70 dds. La EUN acumulada fue de 33.59\%. Estos resultados reflejan la 
importancia del uso de la técnica isotópica para trabajos de nutrición vegetal, que permitirán revisar las recomendaciones de manejo de la fertilización nitrogenada para el cultivo de maíz de altura.

\section{RECONOCIMIENTO}

Los autores agradecen el apoyo del Dr. Takashi Muraoka, profesor del Centro de Energía Nuclear para la Agricultura (CENA) de Piracicaba, Brasil, quien ayudó con el análisis de los resultados de laboratorio. Esta investigación se ejecutó en el marco del proyecto del Organismo Internacional de Energía Atómica (OIEA) RLA/5/065 "Mejora de los sistemas de producción agrícola mediante la eficacia en el uso de los recursos (ARCAL CXXXVI)". Un resumen de este trabajo se presentó en el I/ Simposio Latinoamericano de Aplicaciones Nucleares en la Agricultura realizado en marzo de 2020 en la ciudad de Quito, Ecuador.

\section{CONTRIBUCIÓN DE LOS AUTORES}

Soraya Alvarado y Franklin Valverde concibieron la investigación y metodología; junto a Rafael Parra desarrollaron y realizaron el trabajo de campo y laboratorio; Yamil Cartagena y José Luis Zambrano realizaron los análisis estadísticos y redactaron el manuscrito. Todos los autores revisaron críticamente el contenido intelectual del manuscrito. 


\section{REFERENCIAS}

[1] ISAAA (International Service for the Acquisition of Agri-biotech Applications) (2014). Nitrogen use efficient biotech crops. Pocket K No. 46. Available at: https://www.isaaa.org/resources/publications/pocketk/46/default.asp

[2] Morales, E., Rubí, M., López, J., Martínez, A., \& Morales, E. (2019). Urea (NBTP) una alternativa en la fertilización nitrogenada de cultivos anuales. Revista Mexicana de Ciencias Agricolas 10 (8): 1875-1886.

[3] Boaretto, A., Muraoka, T., \& Trevelin, P. (2007). Uso eficiente del nitrógeno de los fertilizantes convencionales. Informaciones agronómicas. 13-14.

[4] Llive, F. M. (2016). Vulnerabilidad y dependencia internacional de fertilizantes en el Ecuador. Revista Tecnológica ESPOL, 29(2): $69-88$.

[5] Rouanet, J., Pino, I., A, P., \& Nairo, A. (1999). Eficiencia de uso de fertilizante en el cultivo de trigo. En J. Roaunet, I. Pino, P. A., \& A. Nairo, Nitrógeno en producción de trigo, eficiencia de uso de N-fertilizante por la planta y $\mathrm{N}$-fertilizante por el suelo (págs. 23 - 30). Santiago de Chile.

[6] Motato, N., Pincay, J., Avellán, M., Falcones, M., \& Aveiga, E. (2016). Fertilización del híbrido experimental de maíz INIAP H-603, con base en la eficiencia agronómica del nitrógeno. ESPAMCIENCIA 7 (2): 109-116.

[7] Uhart, S., \& Echeverría, H. (2006). El rol del nitrógeno y el fósforo en la producción de maíz. Diagnóstico de la fertilización nitrogenada y fosforada. Semillas Hibridas Morgan, 5-8-16.

[8] Stewart, W. (2007). Consideraciones en el uso eficiente de nutrientes. Informaciones agronómicas, 67:3.

[9] Cassman, K., Dobermann, A., \& Walters, T. (2002). Agroecosystems, nitrogen use efficiency and nitrogen management. Agronomy and Horticulture Faculty Publications. 2-4.

[10] Barbieri, P., Echeverria, H., Sainz, H., \& Maringolo, M. (2010). Fertilización de maíz con urea de liberación lenta: pérdida por volatilización y eficiencia de uso de nitrógeno. Ciencia del suelo. 28: 57-66.

[11] Guzmán, G., \& Mielgo, A. (2008). Buenas prácticas en producción ecológica. Uso de abonos verdes. Santa Fé de Granada - España: Ministerio de Ambiente, Rural y Marino.

[12] Alvarado, S., Valverde, F., Quishpe, J., \& Parra, R. (2014). Los abonos verdes. Proyecto PIC-12-INIAP-009 "Manejo adecuado de abonos verdes y microorganismos fijadores de nitrógeno dentro de sistemas de producción agroecológicos". Quito.

[13] Clewer, A. G., \& Scarisbrick, D.H. (2001). Practical statistics and experimental design for plant and crop science. John Wiley \& Sons, Ltd. Chichester, England. 331p.

[14] CIMMYT (Centro Internacional de Mejoramiento de Maíz y Trigo) (1995). Manejo de los ensayos e informe de los datos para el Programa de Ensayos Internacionales de Maíz del CIMMYT. Disponible en: https://repository.cimmyt.org/ handle/10883/764

[15] Aguilar-Carpio, C., Salvador Escalante, J. A., Estrada, I. Aguilar Mariscal, J. A. Mejía Contreras, V. F. Conde Martínez, A., \& Trinidad Santos. (2016). Eficiencia agronómica, rendimiento y rentabilidad de genotipos de maíz en función del nitrógeno. Terra Latinoamericana 34: 419-429.

[16] Etchevers, J. (1988). Análisis químico de suelos y plantas. Centro de Edafología, Colegio de Posgraduados. Montecillo, Estado de México.

[17] Caviedes, M. (1986). "INIAP-180": Nueva variedad de maíz de alto rendimiento. INIAP, Estación Experimental Santa Catalina, Programa de Maíz. (Boletín Divulgativo no. 180). Quito, Ecuador. Disponible en: http://repositorio.iniap.gob. ec/jspui/handle/41000/255

[18] Yánez, C., Velásquez, J., Peñaherrera, D., Zambrano, J., Caicedo, M., Heredia, J., Sangoquiza, C., \& Quimbita, A. (2010). Guía de producción de maíz de altura. INIAP, Estación Experimental Santa Catalina, Programa de Maíz. (Guía no. 96). Quito, Ecuador: Disponible en: http://repositorio.iniap.gob.ec/handle/41000/2440

[19] Urquiaga, S. \& Zapata, F. (2000). Manejo eficiente de la fertilización nitrogenada de cultivos anuales en América Latinay el Caribe. Porto Alegre; Génesis; Río de Janeiro. EMBRAPA Agrobiología 110 p. 
[20] Barrie, A. (1991). New methodologies in stable isotope analysis. p. 3-36. Proceedings of Symposium Stable Isotopes in Plant Nutrition, Soil Fertility and Environmental Studies, Vienna. 1-5 0ctober 1991. International Atomic Energy Agency, Vienna, Austria.

[21] Di Rienzo J.A., Casanoves, F., Balzarini, M.G., Gonzáles, L., Tablada, M., \& Robledo, C.W. (2020). InfoStat versión 2020. Centro de Transferencia InfoStat, FCA, Universidad Nacional de Córdoba, Argentina. Disponible en: http://www. infostat.com.ar

[22] García, J., Murillo, B., Nieto, A., Fortis, M., Márquez, C., Castellanos, E., Quiñones, J., \& Avila, N. (2010). Avances en investigación y perspectivas del aprovechamiento de los abonos verdes en la agricultura. Terra Latinoamericana, 28(4), 391-399.

[23] Castro, E., Sierra, A., Mojica, J., Carulla, J., \& Lascano, C. (2016). Uso múltiple de leguminosas como abono verde, en rotación con maíz y heno, para producción de leche. Corpoica Ciencia y Tecnología Agropecuaria. 17. 17. 10.21930/rcta. vol17_num1_art:456.

[24] Martín, G., \& Espinosa, R. (2004). Mineralización del nitrógeno incorporado con los abonos verdes y su participación en la nutrición de cultivos de importancia económica. Cultivos Tropicales. 25. 89-96.

[25] Salazar, E., Beltrán, F., Fortis, M., Leos, J., Cueto, J., \& Vázquez, C. (2003). Mineralización de nitrógeno en el suelo y producción de maíz forrajero con tres sistemas de labranza. Terra. 21:561-567.

[26] Boada, R., \& Espinosa. J. (2016). Factores que limitan el potencial de rendimiento del maíz de polinización abierta en campos de pequeños productores de la Sierra de Ecuador. Revista Siembra 3: 067-082

[27] Zamudio, B., Tadeo, M., Espinosa, A., Martínez, J., Celis, D., Valdivia, R., \& Zaragoza, J. (2015). Eficiencia agronómica de fertilización al suelo de macro nutrimentos en híbridos de maíz. Revista Mexicana de (iencias Agrícolas, 6(7), 15571569.

[28] INAMHI. (Instituto Nacional de Meteorología e Hidrología). (2018). Anuario Meteorológico. Quito: INAMHI. 